\title{
Atlas construction and image analysis using statistical cardiac models
}

\author{
Mathieu De Craene ${ }^{1,2}$, Federico M. Sukno ${ }^{2,1}$, Catalina Tobon-Gomez ${ }^{1,2}$, \\ Constantine Butakoff ${ }^{1,2}$, Rosa M. Figueras i Ventura ${ }^{1,2}$, Corné Hoogendoorn ${ }^{1,2}$, \\ Gemma Piella ${ }^{1,2}$, Nicolas Duchateau ${ }^{1,2}$, Emma Muñoz-Moreno ${ }^{1,2}$, Rafael \\ Sebastian $^{3}$, Oscar Camara ${ }^{1,2}$ and Alejandro F. Frangi ${ }^{1,2,4}$ \\ Center for Computational Imaging \& Simulation Technologies in Biomedicine; \\ ${ }^{1}$ Department of Information and Communication Technologies, Universitat Pompeu \\ Fabra, Barcelona, Spain \\ 2 Biomedical Research Networking Center in Bioengineering, Biomaterials and \\ Nanomedicine (CIBER-BBN), Barcelona, Spain \\ ${ }^{3}$ Department of Computer Science, Universitat de Valencia, Valencia, Spain \\ ${ }^{4}$ Institució Catalana de Recerca i Estudis Avançats (ICREA), Spain.
}

\begin{abstract}
This paper presents a brief overview of current trends in the construction of population and multi-modal heart atlases in our group and their application to atlas-based cardiac image analysis. The technical challenges around the construction of these atlases are organized around two main axes: groupwise image registration of anatomical, motion and fiber images and construction of statistical shape models. Applicationwise, this paper focuses on the extraction of atlas-based biomarkers for the detection of local shape or motion abnormalities, addressing several cardiac applications where the extracted information is used to study and grade different pathologies. The paper is concluded with a discussion about the role of statistical atlases in the integration of multiple information sources and the potential this can bring to in-silico simulations.
\end{abstract}

\section{Introduction}

Four dimensional medical images become ubiquitous as routine diagnostic tool. They also are very important research instruments that allow understanding normal and diseased conditions, and grading the severity or stages of a disease. The variety of clinically available image modalities base their acquisition on wide physical phenomena. Hence, each modality offers the possibility to observe the object of interest from a distinct perspective, sometimes unique, sometimes complementary to other related imaging examinations. Clinicians extract and complement information from different studies to form a mental model, in three or four dimensions, which may allow them to classify each patient's pathology. Nonetheless, parameter values can not be regarded as comparable since each of them is measured under distinct principles. Ideally, information should be extracted from the various sources through the application of methodologies that 
This a pre-print version.

The final document is available at http://www.springerlink.com

minimize the bias towards the specific processing chain and, instead, enhance the fact that there is a physical or physiological complementarity of the underlying information sources. Statistical atlases and models are a recognized paradigm to integrate observations into quantitative, self-consistent and comprehensive descriptions [52]. This is particularly challenging in the context of cardiac imaging, since both shape and motion variability must be taken into account.

\subsection{Atlas challenges and evolution}

Historically, anatomical atlases aimed at providing a standard reference frame for comparing patients in a common space. They were obtained through the detailed segmentation of a single subject, considering this subject to be representative of the standard anatomy. Over the years, atlases have evolved to encode, on top of the template anatomy, the variability within a population. In this context, groupwise alignment of a population generated considerable research interest $[6,14,50]$. Several challenges were tackled to extend atlas construction algorithms to large populations, such as the automatic detection of cases with incorrectly parameterized anatomy (outliers) [4,8], the design of flexible registration techniques that can solve for small and large transformations and produce smooth and invertible transformations without compromising registration accuracy $[42,51]$ and the appropriate selection of the reference instance(s) [6]. Recent advances on this latter issue are presented together with some of our recent work on this matter in Section 2.1.

In parallel to extending traditional atlases towards population atlases, an additional challenge is to integrate multi-scale and heterogeneous information. In cardiac studies, this information can consist of myocardial motion and deformation, perfusion, and fiber orientation, to name just a few. Issues related to the construction of motion and fiber atlases are discussed in Sections 2.2 and 2.3.

After bringing into correspondence multi-modal data from all subjects, the anatomical variability captured in the population needs to be projected on a set of basis functions, giving a more compact representation of this variability. A widespread option is to construct a statistical shape model $[9,10]$ based on Principal Component Analysis (PCA). Statistical shape models have been applied to all major cardiac imaging modalities, with encouraging results for the quantification of cardiac function [21,36,47] (Section 3).

Alternatives to the PCA representation focus on localized components such as Independent Component Analysis (ICA) [45], sparse component analysis [44], or physiologically inspired modes like thickening or twisting [41].

\subsection{Some applications of cardiac atlases}

The application of atlases to cardiac studies can be articulated around three main axes: (1) the automatic extraction of patient-specific biomarkers, (2) the integration of multi-modal data into a unified space for visualization purposes and (3) the generation of patient-specific models for personalized simulation of alternative treatment scenarios. 
This a pre-print version.

The final document is available at http://www.springerlink.com

Section 4 presents three possible strategies for extracting probabilistic biomarkers detecting and quantifying pathological shape or motion abnormalities. By probabilistic biomarkers, we mean indexes that compare the value of a given parameter representing local anatomy and function towards a population atlas and output a statistical significance test of observing this same value within the population. The first example of biomarker we describe in this paper focus on local myocardial thickness for differentiating hypertrophic myopathy and hypertensive diseases (Section 4.1). Another example of biomarker concerns the local quantification of abnormality in myocardial velocities, and their application to the quantification of cardiac dyssynchrony in the context of cardiac resynchronization therapy (Section 4.2). The last example of biomarker presented in this paper integrates local shape and motion information using bilinear models for the integrated detection of shape and motion abnormalities (Section 4.3). Perspectives in the second and third axes are briefly given in Section 5 .

\section{Image registration for automated construction of cardiac atlases}

Image-based inter-subject registration has established itself as a corner stone for populational atlas construction. Its main function is to bring into correspondence the anatomy of large databases of $3 \mathrm{D}$ or $3 \mathrm{D}+\mathrm{t}$ acquisitions. This is a prerequisite for further statistical modeling of the variability included in the population under study.

From the group-wise alignment procedure, the entire population can be encoded in the form of an average exemplar of the anatomy, and a representation of the anatomical variability obtained by analysis of the deformation fields that warp the atlas image to all the sample images in the population [31].

Obtaining the atlas anatomy from large populations has been a central question for ensuring reproducibility and robustness of the atlas construction pipeline. Incorporating the computation of the atlas within the groupwise registration guarantees that the same reference will be obtained by different operators, if there exists a unique solution to the problem (Section 2.1).

The obtained atlas anatomy defines a basic structural layer on which all patients from the population can be mapped and compared. This layer can be seen as a skeleton on which relevant pathological descriptors will be projected. Possible descriptors include, but are not limited to, motion (Section 2.2), strain, perfusion, fibers (Section 2.3) etc.

\subsection{Atlases of cardiac anatomy}

The construction of a statistical atlas of the heart first requires the joint alignment of a population into a Normalized Coordinate System (NCS). In our previous work $[19,31]$, a registration pipeline was proposed to efficiently register large populations and compute the NCS simultaneously. 
This a pre-print version.

The final document is available at http://www.springerlink.com

The complete procedure iterates between registering each subject to the current estimate of the NCS, averaging the set of obtained non-rigid transformations and applying the inverse of this average transformation to the current NCS estimate, as originally proposed by Guimond [20]. The reason for updating iteratively the reference is to remove a potential bias towards an atypical choice of reference within the input population.

Indeed, if the constructed atlas is biased and depicts peculiar anatomical features for the population being studied, there is a risk that when normalizing an individual sample to this space, systematic shape difference to the template anatomy could arise. This, in turn, could further influence statistical analysis [14]. Alternatives for choosing an initial reference include ranking based on groupwise mutual information, where the histograms used are those of the candidate image and the aggregate histogram of the remainder of the population [23]; minimizing the mean square TPS bending energy [32]; and manual selection [33].

\subsection{Atlas of myocardial motion}

The construction of statistical atlases of motion requires to generalize the concepts of aligning large populations of $3 \mathrm{D}$ data to handle the temporal dimensionality of this data, so that motion within each sequence can be integrated in the registration and statistical analysis pipeline. This process can be divided in two steps.

The first step consists in extracting motion from cardiac sequences. Traditionally, this has been estimated by decomposing the problem as a sequential set of pairwise registrations [7], which can be made diffeomorphic (continuous, differentiable and with continuous inverse [49]) in space so that the topology and the orientation of anatomical structures are preserved. However, such a strategy does not enforce temporal consistency, particularly critical when handling spatiotemporal sequences. This constraint was partially addressed by the introduction of a time-continuous transformation [27], although the temporal causality of motion is not completely represented by such a scheme. This can be achieved by extending pairwise diffeomorphic registration approaches as proposed in $[11,12,17]$. In such cases, the total motion field is obtained as the composition of smooth and continuous velocity fields over time, which ensures that motion depends on all previous time points, and is diffeomorphic in both time and space.

The second step addresses normalization of the different sequences to a reference anatomy. A first pipeline adapted to cardiac image sequences was proposed by [35]. The use of diffeomorphic paths was recently proposed for the alignment and the comparison of longitudinal datasets, using parallel transport techniques $[28,39]$, but has not been applied to cardiac images yet. The accuracy of the computed paths can be increased by coupling the inter- and intrasubject registration, as described in [38]. In any of these normalization methods, the transport of the locally computed motion fields to a reference anatomy requires their local reorientation. This operation is generally achieved using a push-forward action on vector fields [40], based on the Jacobian of the mapping to the reference. 


\subsection{Atlas of myocardial fibers}

The use of Diffusion Tensor Imaging (DTI) opens the possibility to extend anatomical atlases to model the myocardial fiber structure, which is essential for building realistic models of the electro-mechanical contraction of the heart. Traditionally, models of the muscle tissue have been based on histological studies, but in recent years, DTI has been used to analyze fiber orientation [2]. This image modality measures the molecular diffusion in tissues, by the estimation of a diffusion tensor (DT) at each voxel, that describes direction, magnitude and anisotropy of the diffusion [5]. Since molecular diffusion is constrained by the cell membranes, the principal direction of diffusion provides an estimation of the fiber orientation. From the eigen analysis of the DT, the principal direction can be computed (Fig. 1.a) and tractography algorithms [29] can be applied to DTI data sets to visualize fibers in $3 \mathrm{D}$, as shown in Fig. 1.

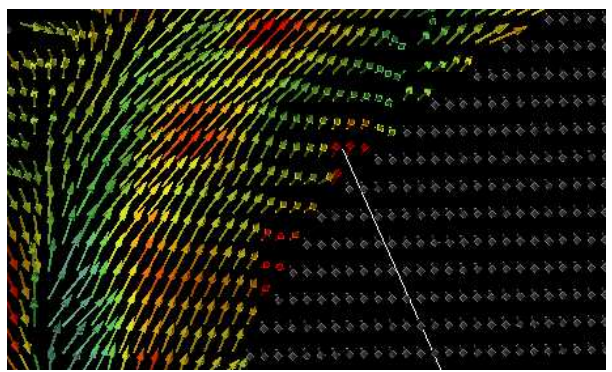

(a)

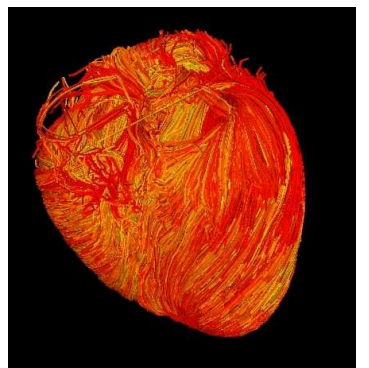

(b)

Fig. 1. a) Detail of the fiber orientation in the septal wall of the left ventricle; b) $3 \mathrm{D}$ reconstruction of fibers in the myocardium computed from DTI.

To remove individual variation and to build models of the fiber distribution, images are aligned to a reference and then averaged. This spatial normalization must be performed with DTI-specific registration methods, as the use of algorithms proposed for other modalities require their adaptation to deal with the tensorial nature of the data. The main issues are the definition of the similarity metric that is optimized along the registration procedure, and the need for warping tensors coherently with the geometrical transformation of the image. In Muoz-Moreno \& Frangi [30], we propose a similarity metric related to different features of the diffusion as orientation, shape and magnitude to perform the registration. Initial results point out the advantages of these specific metrics. Since tensors are related to the fiber orientation, if this orientation changes due to a rotation of the registered volume, tensors should be reoriented to remain aligned with the fiber structures [1].

On the other hand, since atlas construction involves averaging and statistical analysis over the aligned data, the definition of tensor calculus frameworks 
This a pre-print version.

The final document is available at http://www.springerlink.com

for averaging the DT is another challenging topic. In [30], a framework to compute the average between tensors is also described to obtain more accurate fiber models.

\section{Automatic construction of Statistical Shape Models}

A very extended strategy to represent objects in medical imaging and other fields, where there is a necessity to extract the geometry of objects from an image, is to employ several keypoints or landmarks distributed along the object's boundary [15]. Among techniques using such representation we are especially interested in Active Shape Models (ASMs) [10], as they allow for a highly automated processing. They constitute a generative model-based approach in which a priori information of the class of objects of interest is encoded into a Point Distribution Model (PDM), which is constructed by applying Principal Component Analysis (PCA) to the set of aligned training shapes. As it can be identified from the name of PDM, it characterizes the distribution of landmarks, therefore it is imperative that the shapes in the training set have a consistent landmarking strategy so that every landmark is placed in the same spot in all the instances of the object. This is often obtained through manual annotation. While this task is possible in $2 \mathrm{D}$, where a hundred landmarks are usually more than enough, its extension to volumetric 3D data is nearly unfeasible. The possibility to construct large population atlases (Section 2), which relate every image to the template through invertible deformation fields can drastically simplify the task: it is enough to annotate the template image and propagate the annotations to the remaining dataset using the inverse transform $[19,31]$. As a result, this framework can provide highly consistent annotations for large sets of images with minimal amount of manual labor.

To extract the geometry from an image, the PDM has to be fit to it. This is taken care of by the other component of the ASM - local intensity models. As opposed to the cardiac geometry, that is the same independently of the image modality, the intensity models do depend on the imaging technique. Extracting the geometry from these modalities would require creating a training set for each of them. To overcome this difficulty in an automatic manner, we investigated the possibility of using synthetic datasets training intensity models [47].

A number of medical image acquisition simulation tools have been developed for virtually all major medical image modalities [24, 25,43]. They usually require as input a 3D labeled image, which provides the anatomy and the tissue properties. The labeled image can be generated from the PDM itself, or from computer phantoms like 4D-XCAT [43], with the enormous advantage of an accurate knowledge of the geometry that will be embedded in the generated images. This approach to train the intensity model, eliminates the costly and expert dependent process of manual delineation, and allows constructing arbitrarily large training sets, wherefrom the intensity models can be learned in a completely automatic manner. 
This a pre-print version.

The final document is available at http://www.springerlink.com

\section{Identification and analysis of abnormal patterns}

\subsection{Morphological analysis of the left ventricle}

Although most of the contemporary cardiac imaging modalities provide high resolution 3D images, cardiac morphological analysis at clinical level is performed with a few 2D distance measurements. These distance measurements are used to categorize the pathology of the patient. For instance, an infarcted patient presents a localized thin wall; a dilated patient presents increased left ventricular diameter and overall thin walls; a hypertrophic patient presents either localized or overall thick walls. Recent studies have explored the use of novel geometric indices for diagnosis [3, 26]. Ardekani et al. [3] used PCA to analyze shape differences between a test subject and an average heart to classify ischemic versus non-ischemic dilated cardiomyopathy. Kown et al. [26] analyzed the relationship between the aortic root angle and the degree of left ventricular outflow tract obstruction in hypertrophic patients.

An atlas based segmentation approach allows for this sort of 3D morphological analysis. However, the optimal descriptor that characterizes a pathology (or a phenotype) must be identified. For instance, for left ventricular hypertrophy the expected optimal descriptor is wall thickness (WT). Unfortunately, WT values may vary greatly from normal (WT $\leq 12 \mathrm{~mm}$ ), to mild hypertrophy (WT $\leq 16 \mathrm{~mm}$ ), to severe hypertrophy (WT $\leq 30 \mathrm{~mm}$ ). This creates a phenotypic overlap, which clearly complicates the characterization of each condition [37].

A proper analysis of the spatial distribution of the hypertrophy can shed some light on this issue. Such an analysis can be performed thanks to the 3D atlasbased segmentation, which assures topology consistency among all datasets. Therefore, we can compute wall thickness measurements per vertex and patient and analyze the thickness variability in our population. In a recent study, we investigated this possibility [48]. The methodology was tested in fifty three subjects: 18 patients with hypertrophic cardiomyopathy, 13 patients with hypertensive heart disease and 22 sedentary subjects. Control subjects were successfully classified in $96 \%$ of the cases. The classification of each hypertrophic phenotype was correct in $90 \%$ of the cases.

\subsection{Atlas-based motion indexes for the characterization of septal flash in CRT patients}

In Duchateau et al. [16], we proposed an atlas-based pipeline for the analysis of abnormal septal motion patterns in cardiac resynchronization therapy (CRT) patients from $2 \mathrm{D}$ echocardiographic images. A motion atlas was built from a population of 21 volunteers and locally represents the average septal velocity in the atlas space of coordinates. The variability around this average is encoded by the covariance matrix of the local velocity distribution. This gives a compact representation of healthy motion and allows for the computation of a local index of abnormality based on a statistical distance from the atlas (Mahalanobis distance and associated $p$-value). This index encodes the probability of locally 

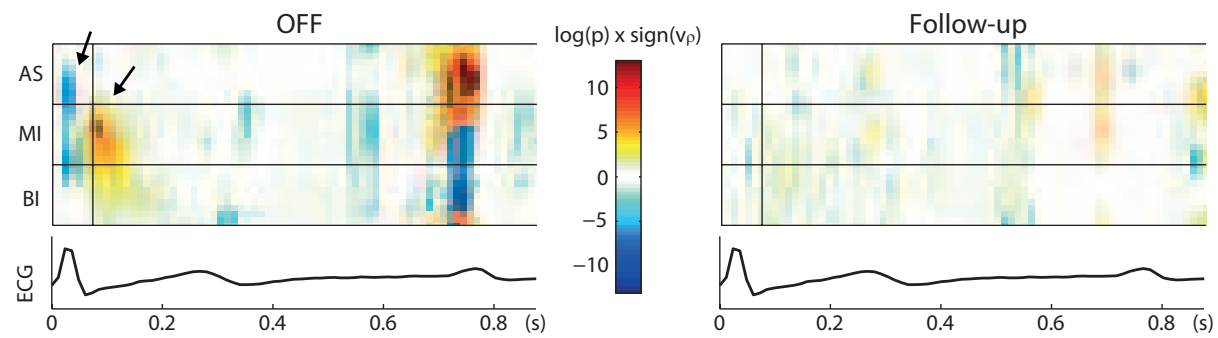

Fig. 2. Abnormality maps for septal motion, before CRT and at 12 months follow-up. The correction of the septal flash pattern (black arrows) is accurately located and quantified through this mode of representation.

observing a motion pattern on an individual, given the variability expected from a healthy population. Low $p$-values indicate high degree of abnormality.

These $p$-values are available at any point in time and space, which allows convenient representation for clinical studies: an abnormality map can be plotted on top of the gray level image at each time point for accurately relating the localization of motion abnormalities with the cardiac anatomy. A second alternative consists in representing a spatiotemporal map of abnormality, as illustrated in Fig. 2. The horizontal axis represents time and the position in the septum (basal inferoseptal [BI], mid inferoseptal [MI], and apical septal [AS]) is used as vertical axis. In this figure, the $p$-value is displayed in logarithmic scale and multiplied by the sign of the radial velocity to enlighten abnormal inward and outward motion patterns.

The potential of this new index is directly visible in this figure for quantifying changes in myocardial motion induced by CRT. Before the therapy, a fast inward-outward motion pattern known in the literature as septal flash (SF) [34] is observed during the isovolumic contraction period, as indicated by the two arrows. At 12 months follow-up, the spatiotemporal map shows that the abnormality has been totally corrected, which correlates with the clinical response of this patient to therapy. It was demonstrated in [34] that the correction of the SF mechanism was highly predictive of a positive clinical response to CRT.

\subsection{Dimensionality reduction in spatio-temporal cardiac morphological analysis}

Motion analysis is a key element to deal with certain moving organs (such as heart or arterial flow) since many pathologies cannot be identified with static anatomical analysis. This has caused a growing interest in moving sequences of $4 \mathrm{D}$ medical data $(3 \mathrm{D}+$ time), and the need to develop proper tools to analyze these sequences. While traditional shape modeling methods may still be used in these settings (e.g. by processing every temporal frame), they are not designed to handle (nor to take advantage of) the temporal component. 
This a pre-print version.

The final document is available at http://www.springerlink.com

The ideal model should result from one single modeling step, where the two different sources of variation (patient anatomy and motion) are recognized and incorporated in a compact, meaningful way. In a recent work [22], we used bilinear models to establish an anatomy/motion factorization on cardiac shapes.

Bilinear and multilinear models $[13,46]$ are multi-dimensional generalizations of singular value decomposition, where two different types of information can be represented in a bilinear basis through two sets of coefficients. These models allow to create one set of coefficients for changes due to motion and one set of coefficients for changes due to patient anatomy, but where all the coefficients are related to the same basis and obtained simultaneously. They have been effectively used to separate anatomical information of a patient from its motion behavior, giving very compact representation of both anatomy and motion $[18,22]$. This kind of models can be applied to the template mesh, obtained with an automated segmentation approach based on atlases or using a statistical method. Its direct use in image segmentation has also been demonstrated [53].

The compact and independent representation of anatomical and motion variations in bilinear models can be used to efficiently apply movement of a given patient, obtained through a dynamic modality such as magnetic resonance (MRI) or ultrasound (US), to still images, as shown in the experiments performed in $[18,22]$, where motion is extrapolated from only the first images of the sequence. This can be used as interventional aid, giving the possibility of moving the reference image used to implant devices in the heart according to specific patient motion coefficients, and thus improving their precision in localizing the implant zone.

\section{Other applications of statistical cardiac models: multimodal integration of structural, sub-structural and functional information}

Multimodal imaging can help clinicians by providing integrated information, beyond the limitations of single modalities. On the other hand, multimodal data provides different (and often incomplete) information layers from the same organ. Hence, the data fusion is often complicated and many times is performed implicitly in the clinician's mind, with evident shortcomings. In this context, methods based on statistical atlases provide a natural way of integration. The presence of a reference anatomy serves as a template where information can be mapped. As explained in the previous sections, multimodal information can include geometric measurements, deformation/motion patterns, myocardial fiber structure, tissue elastic and activation properties, etc.

Fig. 3 illustrates this concept: a four chamber model of the heart in which multimodal information has been reconstructed and integrated from the most appropriate modality (CT for atrial geometry; structural MRI for ventricular geometry; DT-MRI for fiber orientation; and electroanatomical mapping, EPM for electrical activation). The integration of multiple sources of information into the same anatomical reference will allow the investigation of the interplay among 
various parameters associated with a disease condition. Key elements for data integration are the accuracy of the correspondences, identified either by registration (Section 2) or by model-to-image adaptation (Section 3), the repeatability of the measurements and the degree of automation. However, none of this can be considered solved problems, except for very specific situations.

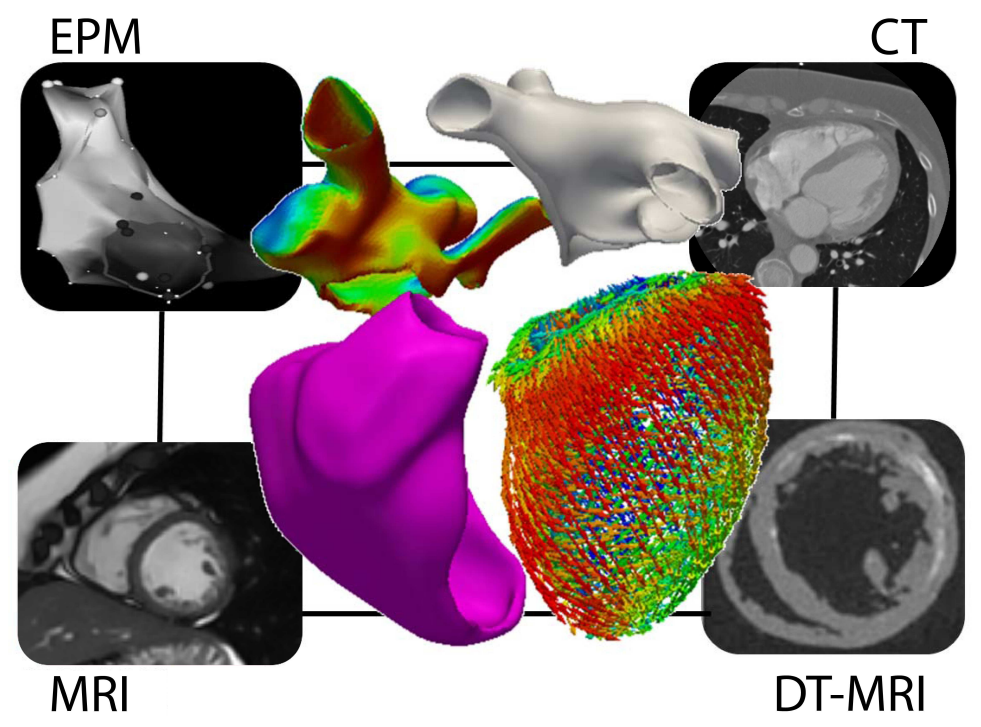

Fig. 3. A four chamber model of the heart in which multimodal information has been reconstructed and integrated from the most appropriate modality

Besides its value for image analysis and image-based diagnosis, the integration of multimodal information is required for the personalization and validation of computational physiology models. The use of simulations to model the multiphysics (mechanics, electrophysiology, fluid) mechanisms that govern the heart behaviour can help to better understand pathological processes affecting the heart function as well as providing additional information to the clinician for improved diagnosis and optimized interventional planning. Hence, the resulting in silico simulations are more realistic when considering multi-scale data that could be available in a statistical atlas, including information extracted from images or models of substructural information such as myocardial fibres or the semi-automatic generation of Purkinje networks generated semi-automatically as proposed by Zimmerman et al. [54]. Finally, simulation results can also be incorporated into the statistical atlas as a new layer of information since computational models can provide insights about non-observable parameters such as contractility or apparent conductivity maps. 
This a pre-print version.

The final document is available at http://www.springerlink.com

\section{Conclusions}

Computer-aided diagnosis can be thought of as finding measurements or patterns that significantly deviate from normality and exhibit good correlation with pathological states. Statistical atlases build upon this paradigm and aim at establishing a common coordinate frame to facilitate comparison and statistical analysis across populations. The key element is that atlas construction allows to establish dense correspondences between the reference and all datasets involved in the process. Therefore, it is possible to perform measurements that are consistent for a population of individuals, or even fuse the information provided by different image modalities to obtain an augmented representation. This augmented representation is expected to aid the diagnosis, by providing a holistic vision of the patient's condition and ease the prediction of the likely treatment outcome.

\section{Acknowledgements}

This work has been funded by the Integrated Project euHeart (FP7/ICT-2007224495) in the European Commissions 7th Framework Programme, and by the Spanish Ministry of Innovation and Science through grant TIN2009-14536-C0201, Plan E and FEDER, and through the cvREMOD project (CEN-20091044) under the CENIT programme of the Industrial and Technological Development Center (CDTI).

\section{References}

[1] D. Alexander et al. Spatial transformations of diffusion tensor magnetic resonance images. IEEE Trans. Med. Imag., 20(11):1131-1139, 2001.

[2] R. H. Anderson et al. The three-dimensional arrangement of the myocytes in the ventricular walls. Clin. Anat., 22:64-76, 2009.

[3] S. Ardekani et al. Computational method for identifying and quantifying shape features of human left ventricular remodeling. Ann Biomed Eng, 37(6):1043-1054, 2009.

[4] J. Ashburner and K. J. Friston. Voxel-based morphometry - the methods. NeuroImage, 11(6):805-821, 2000.

[5] P. J. Basser et al. Estimation of the effective self-diffusion tensor from the NMR spin echo. J. Magn. Reson. Ser. B, 103(3):247-254, 1994.

[6] D. J. Blezek and J. V. Miller. Atlas stratification. Med. Image Anal., 11(5):443457, 2007.

[7] R. Chandrashekara et al. Analysis of 3-D myocardial motion in tagged MR images using nonrigid image registration. IEEE Trans. Med. Imag., 23(10):1245-1250, 2004 .

[8] O. Commowick et al. Detection of DTI white matter abnormalities in multiple sclerosis patients. MICCAI, LNCS 5241, pp 975-982, 2008.

[9] T. F. Cootes and C. J. Taylor. Anatomical statistical models and their role infeature extraction. Brit. J. Radiol., 77:S133-S139, 2004. 
[10] T. F. Cootes and C. J. Taylor. Statistical models of appearance for computer vision. Tech. Rep., University of Manchester, UK, 2004.

[11] M. De Craene et al. Temporal diffeomorphic free-form deformation for strain quantification in 3D-US images. MICCAI 2010 (in press).

[12] M. De Craene et al. Large diffeomorphic FFD registration for motion and strain quantification from 3D-US sequences. FIMH, LNCS 5528, pp 437-446, 2009.

[13] L. De Lathauwer et al. A multilinear singular value decomposition. SIAM J. Matrix Anal. A., 21(4):1253-1278, 2000.

[14] J. Diedrichsen. A spatially unbiased atlas template of the human cerebellum. NeuroImage, 33(1):127-138, 2006.

[15] I. L. Dryden and K. V. Mardia. Statistical Shape Analysis. Wiley, 1998.

[16] N. Duchateau et al. Septal flash assessment on CRT candidates based on statistical atlases of motion. MICCAI, LNCS 5762, pp 759-766, 2009.

[17] S. Durrleman et al. Spatiotemporal atlas estimation for developmental delay detection in longitudinal datasets. MICCAI, LNCS 5761, pp 297-304, 2009.

[18] R. M. Figueras i Ventura et al. Bilinear point distribution models for heart motion analysis. ISBI 2010, pp 476-479, 2010.

[19] A. F. Frangi et al. Automatic construction of multiple-object three-dimensional statistical shape models: Application to cardiac modeling. IEEE Trans. Med. Imag., 21(9):1151-1166, 2002.

[20] A. Guimond et al. Average brain models: A convergence study Comput. Vision and Image Understanding, 77(2):192-210, 1999.

[21] J. Hansegård et al. Constrained active appearance models for segmentation of triplane echocardiograms. IEEE Trans. Med. Imag., 26(10):1391-1400, 2007.

[22] C. Hoogendoorn et al. Bilinear models for spatio-temporal point distribution analysis: Application to extrapolation of left ventricular, biventricular and whole heart cardiac dynamics. Int. J. Comput. Vision, 2009. In Press.

[23] C. Hoogendoorn et al. A groupwise mutual information metric for cost efficient selection of a suitable reference in cardiac computational atlas construction. SPIE Med. Im. vol 7623, pp 76231R-76231R-9, 2010.

[24] J.A. Jensen and N.B. Svendsen. Calculation of pressure fields from arbitrarily shaped, apodized, and excited ultrasound transducers. IEEE T. Ultrason. Ferr., 39(2):262-267, Mar 1992.

[25] R. K. Kwan et al. MRI simulation-based evaluation of image-processing and classification methods. IEEE Trans. Med. Imag., 18(11):1085-1097, Nov 1999.

[26] D. H. Kwon et al. Steep left ventricle to aortic root angle and hypertrophic obstructive cardiomyopathy: study of a novel association using three-dimensional multimodality imaging. Heart, 95(21):1784-1791, 2009.

[27] M. J. Ledesma-Carbayo et al. Spatio-temporal nonrigid registration for ultrasound cardiac motion estimation. IEEE Trans. Med. Imag., 24(9):1113-1126, 2005.

[28] M. I. Miller and A. Qiu. The emerging discipline of computational functional anatomy. NeuroImage, 45(1, Suppl. 1):S16-S39, 2009.

[29] S. Mori and P. C. M. van Zijl. Fiber tracking: principles and strategies - a technical review. NMR Biomed., 15:468-480, 2002.

[30] E. Muñoz Moreno and A.F. Frangi. Spatial normalization of cardiac diffusion tensor imaging for modeling the muscular structure of the myocardium. In ICIP 2010 (in press).

[31] S. Ordás et al. A statistical shape model of the heart and its application to model-based segmentation. SPIE Med. Im. vol. 6511, pp 6511K, 2007.

[32] H. Park et al. Least biased target selection in probabilistic atlas construction. MICCAI, LNCS 3750, pp 419-426, 2005. 
[33] H. Park et al. Construction of an abdominal probabilistic atlas and its application in segmentation. IEEE Trans. Med. Imag., 22(4):483-492, 2003.

[34] C. Parsai et al. Toward understanding response to cardiac resynchronization therapy: left ventricular dyssynchrony is only one of multiple mechanisms. Eur. Heart J., 30(8):940-949, 2009.

[35] D. Perperidis et al. Spatio-temporal free-form registration of cardiac MR image sequences. Med. Image Anal., 9(5):441-456, 2005.

[36] J. Peters et al. Optimizing boundary detection via simulated search with applications to multi-modal heart segmentation. Med. Image Anal., 14:70-84, 2010.

[37] S. E. Petersen et al. Differentiation of athlete's heart from pathological forms of cardiac hypertrophy by means of geometric indices derived from cardiovascular magnetic resonance. J. Cardiovasc. Magn. Reson., 7(3):551-558, 2005.

[38] J.-M. Peyrat et al. Registration of 4D cardiac CT sequences under trajectory constraints with multichannel diffeomorphic demons. IEEE Trans. Med. Imag., 29(7):1351-1368, 2010.

[39] A. Qiu et al. Time sequence diffeomorphic metric mapping and parallel transport track time-dependent shape changes. NeuroImage, 45(1, Sup. 1):S51-S60, 2009.

[40] A. Rao et al. Spatial transformation of motion and deformation fields using nonrigid registration. IEEE Trans. Med. Imag., 23(9):1065-1076, 2004.

[41] E. W. Remme et al. Extraction and quantification of left ventricular deformation modes. IEEE Trans. Biomed. Eng., 51(11):1923-1931, 2004.

[42] D. Rueckert et al. Diffeomorphic registration using B-splines. MICCAI, LNCS 4191, pp 702-709, 2006.

[43] W. P. Segars et al. Realistic CT simulation using the 4D XCAT phantom. Med. Phys., 35(8):3800-3808, 2008.

[44] K. Sjöstrand et al. Sparse decomposition and modeling of anatomical shape variation. IEEE Trans. Med. Imag., 26(12):1625-1635, 2007.

[45] A. Suinesiaputra et al. Automated detection of regional wall motion abnormalities based on a statistical model applied to multi-slice short-axis cardiac MR images. IEEE Trans. Med. Imag., 28(4):595-607, 2009.

[46] J. B. Tenenbaum and W. T. Freeman. Separating style and content with bilinear models. Neural Comput., 12(6):1247-1283, 2000.

[47] C. Tobon-Gomez et al. Automatic construction of 3D-ASM intensity models by simulating image acquisition: Application to myocardial gated SPECT studies. IEEE Trans. Med. Imag., 27(11):1655-1667, 2008.

[48] C. Tobon-Gomez et al. 3D mesh based wall thickness measurement: identification of left ventricular hypertrophy phenotypes. In EMBS 2010 (in press).

[49] A. Trouvé. Diffeomorphisms groups and pattern matching in image analysis. Int. J. Comput. Vision, 28(3):213-221, 1998.

[50] C. J. Twining and S. Marsland. Constructing an atlas for the diffeomorphism group of a compact manifold with boundary, with application to the analysis of image registrations. J Comput. Appl. Math., 222(2):411-428, 2008.

[51] T. Vercauteren et al. Non-parametric diffeomorphic image registration with the demons algorithm. MICCAI, LNCS 4792, pp 319-326, 2007.

[52] A. A. Young and A. F. Frangi Computational cardiac atlases: from patient to population and back. Experimental Physiology, 94(5):578-596, 2009.

[53] Y. Zhu et al. Segmentation of the left ventricle from cardiac MR images using a subject-specific dynamical model. IEEE Trans. Med. Imag., 29(3):669-687, 2010.

[54] V. Zimmerman et al. Modeling the Purkinje conduction system with a non deterministic rule based iterative method. IEEE Computers in Cardiology, vol 36, pp 461-464, 2009. 